\title{
Expression of Killer Immunoglobulin Receptor Genes among HIV-Infected Individuals with Non-AIDS Comorbidities
}

\author{
Farouk F. Abou Hassan, ${ }^{1}$ Mirna Bou Hamdan, ${ }^{1}$ Khalil El Asmar, ${ }^{2}$ and Nada M. Melhem (D) \\ ${ }^{1}$ Medical Laboratory Sciences Program, Division of Health Professions, Faculty of Health Sciences, American University of Beirut, \\ Beirut, Lebanon \\ ${ }^{2}$ Department of Epidemiology and Population Health, Faculty of Health Sciences, American University of Beirut, Beirut, Lebanon
}

Correspondence should be addressed to Nada M. Melhem; melhemn@aub.edu.lb

Received 29 July 2021; Revised 1 December 2021; Accepted 17 December 2021; Published 12 January 2022

Academic Editor: Jixin Zhong

Copyright (C) 2022 Farouk F. Abou Hassan et al. This is an open access article distributed under the Creative Commons Attribution License, which permits unrestricted use, distribution, and reproduction in any medium, provided the original work is properly cited.

\begin{abstract}
Combined antiretroviral therapy (cART) increased the life expectancy of people living with HIV (PLHIV) and remarkably reduced the morbidity and mortality associated with HIV infection. However, non-AIDS associated comorbidities including diabetes, hypertension, hyperlipidemia, and cardiovascular diseases (CVD) are increasingly reported among PLHIV receiving cART. Killer cell immunoglobulin receptors (KIRs) expressed on the surface of natural killer (NK) cells have been previously implicated in controlling HIV disease progression. The aim of this study is to investigate the role of KIRs in developing nonAIDS associated comorbidities among PLHIV. Demographic and behavioral data were collected from voluntary participants using a standardized questionnaire. Whole blood samples were collected for KIR genotyping. Hypertension (29.5\%) and hyperlipidemia (29.5\%) followed by diabetes (23.7\%) and CVD (9.7\%) were mainly reported among our study participants with higher rate of comorbid conditions observed among participants $>40$ years old. The observed KIR frequency (OF) was $\geq 90 \%$ for inhibitory KIR2DL1 and KIR3DL1, activating KIR2DS4 and the pseudogene KIR2DP1 among study participants. We detected significant differences in the expression of KIR3DS4 and KIR3DL1 $(p=0.038)$ between diabetic and nondiabetic and in the expression of KIR2DL3 between hypertensive and normotensive HIV-infected individuals $(p=0.047)$. Moreover, KIR2DL1 and KIR2DP1 were associated with significantly reduced odds of having CVD (OR 0.08; 95\% CI: 0.01-0.69; $p=0.022$ ). Our study suggests the potential role of KIR in predisposition to non-AIDS comorbidities among PLHIV and underscores the need for more studies to further elucidate the role of KIRs in this population.
\end{abstract}

\section{Introduction}

The use of combined antiretroviral therapy (cART) significantly reduced the morbidity and mortality associated with human immunodeficinecy virus (HIV) infection [1]. The former contributed to an increase in the life expectancy of people living with HIV (PLHIV) approaching that of HIVnegative individuals [2-4]. Consequently, the global proportion of people aging with HIV was estimated to reach $21 \%$ [5]. These data suggest a demographic shift affecting HIV management and care. With the increasing proportion of people living and aging with HIV, non-AIDS (acquired immunodeficiency syndrome) comorbidities have been increasingly reported among treated PLHIV leading to an increased number of deaths exceeding those of AIDSrelated deaths [6-9]. These comorbidities include cardiovascular disease (CVD) [10-15], liver disease [16, 17], renal disease $[11,14,18]$, diabetes $[10-13,15,19]$, and neurocognitive abnormalities [20,21], as well as non-AIDS defining malignancies including liver, brain, anal, and lung cancers [22, 23]. While biological aging was suggested to start earlier among HIV infected individuals ( 55 vs. 65 years) [24], the subsequent pathway leading to disease manifestation among treated and aging PLHIV is not fully understood.

Killer cell immunoglobulin receptors (KIRs) are highly polymorphic type 1 transmembrane glycoproteins expressed on the surface of natural killer (NK) cells [25]. The balance 
between the inhibitory and activating signals received from KIRs regulates the function of NK cells. Genes encoding KIRs are located in the leukocyte receptor complex (LRC) on chromosome 19q13.4 [26]. There are 16 recognized genes that encode for KIR of which 7 are activating (KIR2DS1, KIR2DS2, KIR2DS3, KIR2DS4, KIR2DS5A, KIR2DS5B, and KIR3DS1), 8 are inhibitory (KIR2DL1, KIR2DL2, KIR2DL3, KIR2DL5A, KIR2DL5B, KIR3DL1, KIR3DL2, and KIR3DL3), and one (KIR2DL4) predominantly activating but can transmit inhibitory signals [27]. Two KIR haplotypes are defined in humans: haplotype A and haplotype B [27, 28]. While haplotype A encodes inhibitory receptors (KIR2DL1, KIR2DL3, KIR3DL1, KIR3DL2, KIR3DL3, KIR2DP1, KIR3DP1, and KIR2DL4) and one activating receptor KIR2DS4, haplotype B carries a variety of gene combinations and encodes more activating receptors compared to haplotype A. These include KIR3DL3, KIR2DS2, KIR2DL2, KIR2DL5B (inhibitory) KIR2DS3, KIR2DP1, KIR2DL1, KIR3DP1, KIR2DL4, KIR3DS1, KIR2DL5A (inhibitory), KIR2DS5, KIR2DS1, and KIR3DL2 [29].

Despite the high allelic polymorphism of KIR genes, almost all individuals possess the following genes referred to as framework loci: KIR3DL3, KIR3DP1, KIR2DL4, and KIR3DL2 [27, 28]. Each full-length haplotype is characterized by a centrally located $10-15000$ bp region containing a recombination hotspot [30]. The latter subdivides the haplotype into two regions, namely, proximal or centromeric (Cent) $\left(5^{\prime}\right)$ and distal or telomeric $(\mathrm{Tel})\left(3^{\prime}\right)$ regions which are bound by the framework loci. This recombination hotspot is thus the intermediate region between the centromeric and telomeric regions of the haplotype and bound by the four genes of the framework loci with KIR3DL3 and KIR3DP1 defining the centromere and KIR2DL4 and KIR3DL2 defining the telomere [31]. A centromeric B haplotype is defined by the presence of at least one of the following: KIR2DS2, KIR2DL2, KIR2DL5, KIR2DS3, or KIR2DS5 whereas a centromeric A haplotype by the sole presence of $K I R 2 D L 3$. A telomeric B haplotype is defined by the presence of at least one of the following: KIR3DS1, KIR2DL5, KIR2DS3, KIR2DS5, or KIR2DS1; on the other hand, a telomeric A haplotype is defined by the presence of KIR3DL1 and/or KIR2DS4. Finally, a KIR group A genotype is defined by having centromeric A/A and telomeric A/A; other combinations were denoted KIR group B genotypes [32].

There is scarcity of data on the effect of aging on KIR expression. However, ample data exist on the association between KIR expression and susceptibility, severity, clinical course, or clearance of viral infections (H1N1 2009 influenza, hepatitis $C$ virus, and hepatitis B virus) [33-38] as well as HIV. The proportion of homozygote KIR3DS1 was higher in HIV-exposed uninfected individuals compared to those with primary HIV infection [39], and higher frequencies of KIR3DS1 were associated with low viral load [40]. In addition, HIV-exposed individuals who carry homozygote KIR3DS1 without KIR3DL1 were characterized by a delayed seroconversion compared to exposed individuals with KIR3DL1/S1 heterozygous genotype [41].

Few studies reported the association between KIR gene polymorphism and susceptibility to type 1 diabetes mellitus
(T1DM) [42, 43], hypertension [44], and acute ischemic stroke [45]. KIR2DL2 was suggested as a susceptibility factor whereas KIR2DL1 and KIR2DL5 as protective factors for T1DM. KIR2DL1 and KIR2DS1 were linked to a decreased risk of T1DM among Asians but not Caucasians [43]. Moreover, the expression of KIR2DS5 was suggested as protective against hypertension in a cohort of Chinese Han patients [44]. Higher frequencies of KIR2DL3, KIR2DL5B, KIR2DS2, and KIR2DS4 were reported among patients with ischemic stroke compared to healthy controls [45]. Similarly, the frequency of KIR2DL3 and KIR2DL4 was higher in patients with large artery atherosclerosis compared to controls [45].

The aim of this study is to investigate the relationship between KIR genes and non-AIDS comorbid conditions among a cohort of PLHIV in Lebanon. To our knowledge, there are no data on the relationship between KIR genes expressed among treated HIV-infected individuals and the risk of developing non-AIDS associated comorbid conditions.

\section{Methods}

2.1. Study Design and Ethical Approval. This study was conducted at the American University of Beirut (AUB). Voluntary participants were recruited from three recruitment sites: AUB-Medical Center (AUBMC), Lebanese American University Medical Center-Rizk Hospital (LAUMC-RH), and Soins Infirmiers et Developpement Communautaire (SIDC) - a nongovernmental organization providing health services to HIV-infected individuals. Human subject approval was obtained for this study from the Institutional Review Board (IRB) of AUB and the Lebanese American University (LAU). All participants provided informed consent. A standardized questionnaire was administered to a total of 105 treated adult HIV-infected individuals between November 2018 and December 2019. Demographic and behavioral data including lifestyle, smoking, physical activity, substance use and abuse, coinfections, chronic diseases (cardiovascular disease, hypertension, diabetes, lipid and metabolic disorders, cancer, mental health, and others), first-degree family history (i.e., parents or siblings) of chronic diseases, polypharmacy data, and mental health data were collected.

2.2. DNA Extraction and KIR Gene Genotyping. Out of the 105 voluntary participants, $103 \mathrm{HIV}$-infected individuals provided whole blood samples between November 2018 and December 2019. DNA was extracted using the QIAamp DNA Blood Minikit (Qiagen, Germany) as per manufacturer's instructions. The integrity of the extracted DNA was checked by gel electrophoresis, and its concentration was measured by NanoDrop 2000c (Thermo Fisher Scientific). The purified DNA was stored at $-20^{\circ} \mathrm{C}$. The Polymerase Chain Reaction- (PCR-) based KIR genotyping Sequencespecific Oligonucleotide Hybridization (SSO) Kit (One Lambda, Thermo Fisher, USA) was used to detect the presence and absence of $16 \mathrm{KIR}$ genes, as per manufacturer's instructions. Briefly, $2 \mu \mathrm{l}$ of DNA at an adjusted concentration of $20 \mathrm{ng} / \mu \mathrm{l}$ was used along with the primer sets in a total volume of $20 \mu \mathrm{l}$ per PCR reaction to amplify the alleles. 
All amplifications were performed using SureCycler 8800 (Agilent Technologies) as per the manufacturer's recommendation: 3-minute denaturation step at $96^{\circ} \mathrm{C}$ followed by 5 cycles of $96^{\circ} \mathrm{C}, 60^{\circ} \mathrm{C}$ and $72^{\circ} \mathrm{C}$ for 20 seconds each; 30 cycles of $96^{\circ} \mathrm{C}$ for 10 seconds, $60^{\circ} \mathrm{C}$ for 15 seconds, $72^{\circ} \mathrm{C}$ for 20 seconds; 1 cycle of final elongation step at $72^{\circ} \mathrm{C}$ for 10 minutes; and finally hold at $4^{\circ} \mathrm{C}$. The presence and absence of the following gene loci and variants were determined: KIR2DL1, KIR2DL2, KIR2DL3, KIR2DL4, KIR2DL5, KIR2DS1, KIR2DS2, KIR2DS3, KIR2DS4, KIR2DS5, KIR3DL1, KIR3DL2, KIR3DL3, KIR3DS1, KIR2DP1, and KIR3DP1. Data were acquired using Luminex LABScan $3 \mathrm{D}^{\mathrm{TM}}$ and analyzed using HLA Fusion ${ }^{\mathrm{TM}}$ Research Software Version 6.0.

2.3. Haplotype Classification and KIR Gene Frequencies. KIR haplotypes were defined as previously described [46]. Homozygous A haplotype (AA) was defined by the sole presence of KIR2DL1, KIR2DL3, KIR3DL1, KIR2DS4, KIR2DP1, KIR3DP1, KIR2DL4, KIR3DL2, and KIR3DL3 genes. Other KIR combinations were referred to as Bx haplotype (i.e., BB or BA). The frequency of KIR was calculated by direct count of the observed phenotype and referred to as observed frequency (OF). In addition, the estimated KIR gene frequency $(\mathrm{KF})$ for the putative loci was calculated using the following formula: $\mathrm{KF}=1-\sqrt{ }(1-\mathrm{OF})$ based on the assumption of Hardy-Weinberg equilibrium [47]. We further analyzed participants with Bx genotype based on the expression of different KIR genes combinations. We numbered KIR combinations within the Bx genotype by a number (i.e., $\mathrm{Bx} 1, \mathrm{~B} \times 2, \mathrm{Bx} 3$, etc.) and categorized these into centromeric and telomeric KIR clusters as previously described [30, 46]. The centromeric cluster consists of KIR2DS2, KIR2DL2, KIR2DL5B, and KIR2DS3 combination, while the telomeric half consists of KIR3DS1, KIR2DL5A, KIR2DS5, and KIR2DS1 genes. Bx genotype can exhibit centromeric KIR cluster (KIR2DS2, KIR2DL2, KIR2DL5B, and KIR2DS3 combination), telomeric KIR cluster (KIR3DS1, KIR2DL5A, KIR2DS5, and KIR2DS1 combination) or both [46]. In the analysis, we included the full length KIR2DL5 since we were unable to get the data pertinent to the frequency of KIR2DL5A and KIR2DL5B.

2.4. Statistical Analysis. We compared the frequency of expression of KIR genes and KIR haplotypes between individuals with and without non-AIDS associated comorbid conditions using Fisher's exact test (FET). We performed clustering analysis to identify KIR clusters. We compared the frequency of the latter between individuals with and without comorbidities using FET. We also examined the relationship between KIR expression and clusters of KIR genes versus the presence or absence of non-AIDS-related comorbidities (specifically diabetes, hypertension, hyperlipidemia, and CVD) using multivariate logistic regression model while adjusting for associated risk factors. We corrected for multiple comparisons for post hoc tests using Bonferroni correction; thus, the calculated alpha was 0.05 / $12=0.004$, whereby 12 is the total number of KIR genes
TABle 1: Demographic and clinical characteristics of study participants.

\begin{tabular}{|c|c|c|}
\hline & $n$ & $\%$ \\
\hline \multicolumn{3}{|l|}{ Gender $(n=105)$} \\
\hline Male & 87 & 82.9 \\
\hline Female & 18 & 17.1 \\
\hline \multicolumn{3}{|l|}{ Age in years $(n=105)$} \\
\hline $25-44$ & 42 & 40 \\
\hline $45-59$ & 44 & 41.9 \\
\hline$\geq 60$ & 19 & 18.1 \\
\hline \multicolumn{3}{|l|}{ Sexual history $(n=102)$} \\
\hline Heterosexual & 54 & 52.9 \\
\hline MSM & 33 & 32.4 \\
\hline Bisexual & 14 & 13.7 \\
\hline \multicolumn{3}{|l|}{ HIV route of transmission $(n=105)$} \\
\hline Unprotected sex & 72 & 68.6 \\
\hline Shared needle/syringe & 7 & 6.7 \\
\hline Others & 7 & 6.7 \\
\hline \multicolumn{3}{|l|}{ Duration of HIV infection $(n=103)$} \\
\hline$<5$ years & 9 & 10.3 \\
\hline $5-10$ years & 40 & 38.8 \\
\hline$>10$ years & 54 & 52.4 \\
\hline \multicolumn{3}{|l|}{ Duration of cART $(n=105)$} \\
\hline$<5$ years & 16 & 15.2 \\
\hline $5-10$ years & 42 & 40 \\
\hline$>10$ years & 47 & 44.8 \\
\hline \multicolumn{3}{|l|}{ cART regimen $(n=102)$} \\
\hline NRTI + NNRTI & 47 & 46.1 \\
\hline NRTI + INSTI & 47 & 46.1 \\
\hline Others (PI + NRTI and/or NNRTI) & 8 & 7.8 \\
\hline$\geq 1$ non-AIDS associated comorbidities $(n=105)$ & 44 & 41.9 \\
\hline Hypertension & 31 & 29.5 \\
\hline Hyperlipidemia & 31 & 29.5 \\
\hline CVD & 10 & 9.5 \\
\hline Diabetes & 9 & 8.6 \\
\hline \multicolumn{3}{|l|}{ Non-cART medication $(n=105)$} \\
\hline Antihypertensives & 31 & 29.5 \\
\hline Lipid-lowering agents & 31 & 29.5 \\
\hline Hypoglycaemic agents & 9 & 8.6 \\
\hline
\end{tabular}

MSM: men exclusively having sex with men; cART: combined antiretroviral therapy; NRTI: nucleoside/nucleotide reverse-transcriptase inhibitor; NNRTI: nonnucleoside reverse-transcriptase inhibitor; NNRTI: nonnucleoside reverse transcriptase inhibitor; PI: protease inhibitor.

included in the analysis. All analyses were conducted using STATA SE 13.0.

\section{Results}

3.1. Characteristics of Study Participants. The majority of our participants were $45-59$ years (41.9\%), males $(82.9 \%)$ and heterosexuals $(52.9 \%)$ (Table 1$)$. The majority of our participants contracted HIV-1 and started cART more than 10 


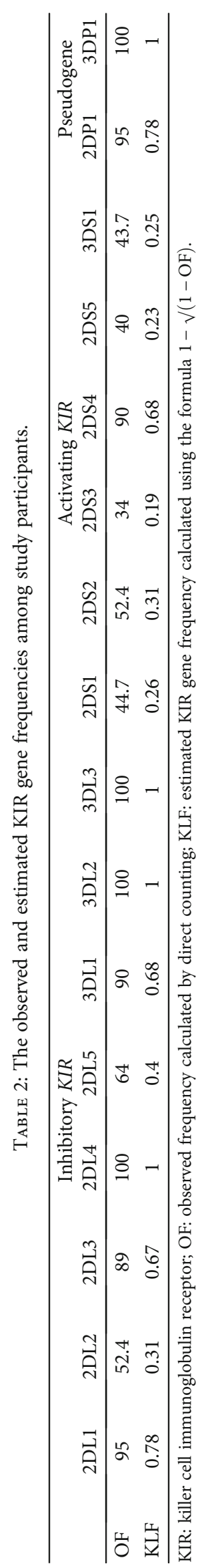




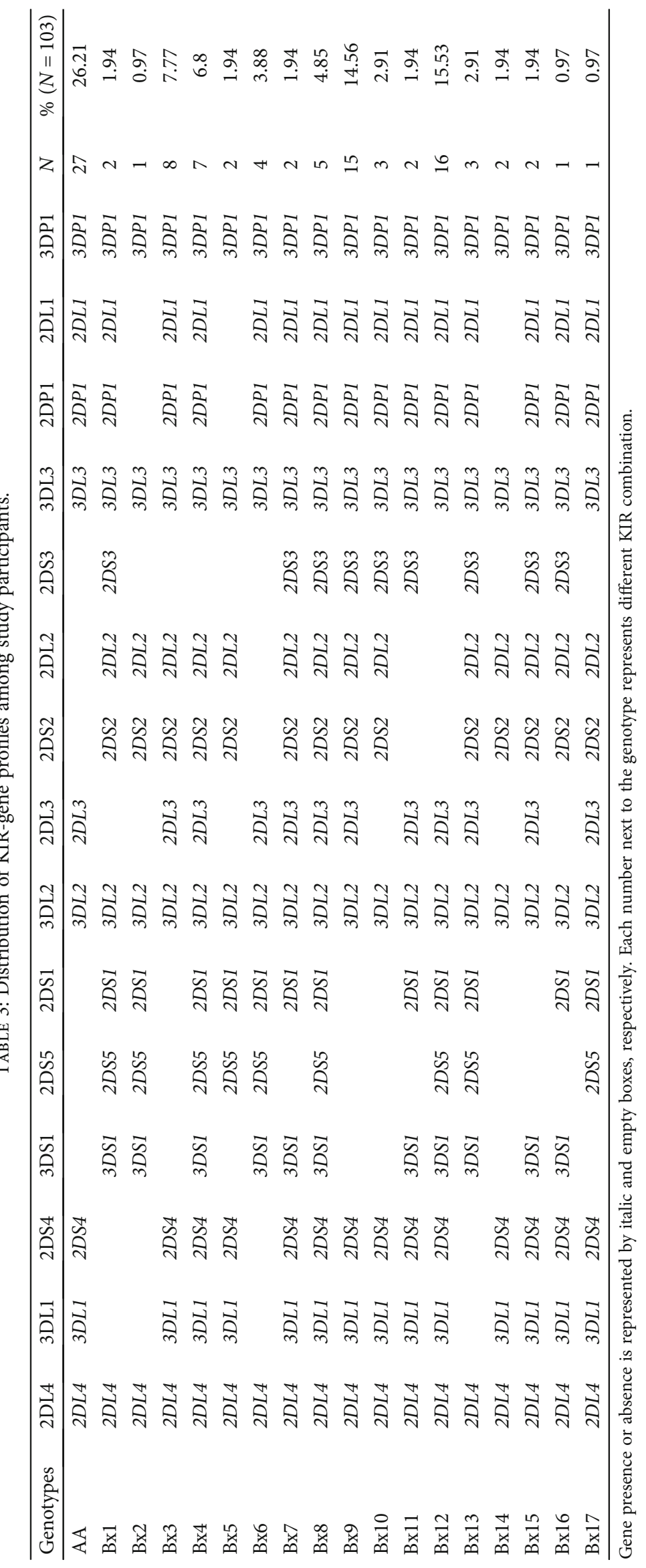


TABLE 4: Centromeric and telomeric gene profiles corresponding to combination of conventional KIR haplotypes.

(a)

\begin{tabular}{|c|c|c|c|c|c|c|c|c|c|c|}
\hline \multicolumn{11}{|c|}{ Centromeric } \\
\hline Genotype & KIR2DL3 & KIR2DS2 & KIR2DL2 & KIR2DS3 & KIR3DL3 & KIR2DP1 & KIR2DL1 & KIR3DP1 & $N$ & $\%(N=103)$ \\
\hline $\mathrm{AA}$ & KIR2DL3 & & & & KIR3DL3 & $K I R 2 D P 1$ & KIR2DL1 & $K I R 3 D P 1$ & 47 & 45.63 \\
\hline Bx1 & & KIR2DS2 & $K I R 2 D L 2$ & KIR2DS3 & KIR3DL3 & KIR2DP1 & KIR2DL1 & KIR3DP1 & 6 & 5.82 \\
\hline $\mathrm{B} \times 2$ & & KIR2DS2 & KIR2DL2 & & KIR3DL3 & & & KIR3DP1 & 1 & 0.97 \\
\hline $\mathrm{B} \times 3$ & KIR2DL3 & KIR2DS2 & KIR2DL2 & & KIR3DL3 & $K I R 2 D P 1$ & $K I R 2 D L 1$ & KIR3DP1 & 16 & 15.53 \\
\hline $\mathrm{Bx} 4$ & & KIR2DS2 & KIR2DL2 & & KIR3DL3 & & & $K I R 3 D P 1$ & 4 & 3.88 \\
\hline Bx5 & KIR2DL3 & KIR2DS2 & KIR2DL2 & KIR2DS3 & KIR3DL3 & $K I R 2 D P 1$ & $K I R 2 D L 1$ & $K I R 3 D P 1$ & 27 & 26.21 \\
\hline Bx6 & KIR2DL3 & & & KIR2DS3 & KIR3DL3 & $K I R 2 D P 1$ & KIR2DL1 & KIR3DP1 & 2 & 1.94 \\
\hline
\end{tabular}

(b)

\begin{tabular}{|c|c|c|c|c|c|c|c|c|c|}
\hline \multicolumn{10}{|c|}{ Telomeric } \\
\hline Genotype & KIR2DL4 & KIR3DL1 & KIR2DS4 & KIR3DS1 & KIR2DS5 & KIR2DS1 & KIR3DL2 & $N$ & $\%(N=103)$ \\
\hline AA & KIR2DL4 & KIR3DL1 & KIR2DS4 & & & & KIR3DL2 & 55 & 53.4 \\
\hline Bx1 & KIR2DL4 & & & KIR3DS1 & KIR2DS5 & KIR2DS1 & KIR3DL2 & 10 & 9.70 \\
\hline $\mathrm{B} \times 2$ & KIR2DL4 & KIR3DL1 & KIR2DS4 & KIR3DS1 & KIR2DS5 & KIR2DS1 & KIR3DL2 & 28 & 27.18 \\
\hline $\mathrm{B} \times 3$ & KIR2DL4 & KIR3DL1 & KIR2DS4 & & KIR2DS5 & KIR2DS1 & KIR3DL2 & 3 & 2.91 \\
\hline $\mathrm{Bx} 4$ & KIR2DL4 & KIR3DL1 & KIR2DS4 & KIR3DS1 & & KIR2DS1 & KIR3DL2 & 3 & 2.91 \\
\hline $\mathrm{Bx} 5$ & KIR2DL4 & KIR3DL1 & KIR2DS4 & KIR3DS1 & & KIR2DS1 & KIR3DL2 & 2 & 1.94 \\
\hline Bx6 & KIR2DL4 & KIR3DL1 & KIR2DS4 & KIR3DS1 & & & KIR3DL2 & 2 & 1.94 \\
\hline
\end{tabular}

Gene presence or absence is represented by italic and empty boxes, respectively. Each number next to the genotype represents different KIR combination.

years ago. NRTI+NNRTI and NRTI+INSTI were the most commonly used drug regimens (Table 1). Forty-two percent of the study participants reported to have one-to-four nonAIDS associated comorbidity with $29.5 \%, 29.5 \%, 9.5 \%$, and $8.6 \%$ suffering from hypertension, hyperlipidemia, CVD, and diabetes, respectively (Table 1). Following the same trend, antihypertensive and lipid-lowering agents were the most commonly reported non-cART medications. Our results showed that the frequency of comorbid conditions increases with age among PLHIV; 19\% of our participants above 40 years old reported one or two comorbid conditions each followed by $10 \%$ and $3 \%$ suffering from 3 and 4 conditions, respectively (Abou Hassan et al. submitted manuscript). Individuals less than 40 years old suffered from less disease conditions with $15 \%$ and $3 \%$ living with one and 2 3 comorbid conditions, respectively.

3.2. KIR Gene and Haplotype Frequencies. We divided the 16 KIR genes into 3 groups: inhibitory KIRs, activating KIRs, and pseudogenes, as previously described [29, 48]. The OF frequency of inhibitory KIR2DL1 and KIR3DL1 and the pseudogene KIR2DP1 were detected in $\geq 90 \%$ among study participants. The OF KIR2DS4 was $90 \%$ while the OF of the remaining activating genes ranged between $34 \%$ and $52 \%$. The estimated KIR gene frequency followed the same order as the OF data (Table 2). As expected, KIR2DL4, $K I R 3 D L 2, K I R 3 D L 3$, and KIR3DP1 were expressed in all individuals. The majority of the study participants $(73.8 \%)$ carried the Bx haplotype while $26 \%$ carried the AA haplotype. We did not detect any significant difference when we compared the frequencies of KIR haplotypes among participants who reported having $\geq 1$ comorbid conditions $(\chi 2, p$ $=1$ ) (data not shown). Based on KIR-gene content, we identified 18 KIR-gene profiles among the study participants: one AA profile and $17 \mathrm{Bx}$ profiles (Table 3 ). The AA KIR profile was predominantly expressed (26\%) followed by $\mathrm{Bx} 12$ (15.5\%) and Bx9 (14.6\%). Moreover, the Cent-AA (45.6\%) followed by Cent-Bx5 (26.2\%) and Bx3 (15.5\%) profiles was most commonly detected (Table 4). On the telomeric side, the Tel-AA (53.4\%), Tel-Bx2 (27.2\%), and Tel-Bx1 $(9.7 \%)$ were the most common profiles. We also detected higher frequency of Cent-B profiles (58\%) than Tel-B profiles $(48 \%)$ (Table 4$)$.

3.3. KIR Genotypes and Non-AIDS Comorbid Conditions. For this analysis and thereafter, we studied the genes with enough variability (KIR2DL1, KIR2DL2, KIR2DL3, KIR2DL5, KIR3DL1, KIR2DS1, KIR2DS2, KIR2DS3, KIR2DS4, KIR2DS5, KIR3DS1, and KIR2DP1). We compared the frequency of $K I R$ genes between participants with and without diabetes, hyperlipidemia, CVD, or hypertension. We detected the following significant differences: KIR3DL1 (inhibitory) and KIR2DS4 (activating) between diabetic and nondiabetic individuals (FET, $p=0.038$ ) and KIR2DL3 (inhibitory) between hypertensives and normotensives (FET, $p=0.047$ ) (Table 5 ). We also detected a significant difference among hypertensive males expressing KIR2DL3 (FET, $p=0.034$ ) (data not shown). Moreover, the expression of KIR2DL1 (FET, $p=0.043$ ), KIR2DP1 (FET, $p$ $=0.043)$, and KIR2DS3 (FET, $p=0.049$ ) was significantly 


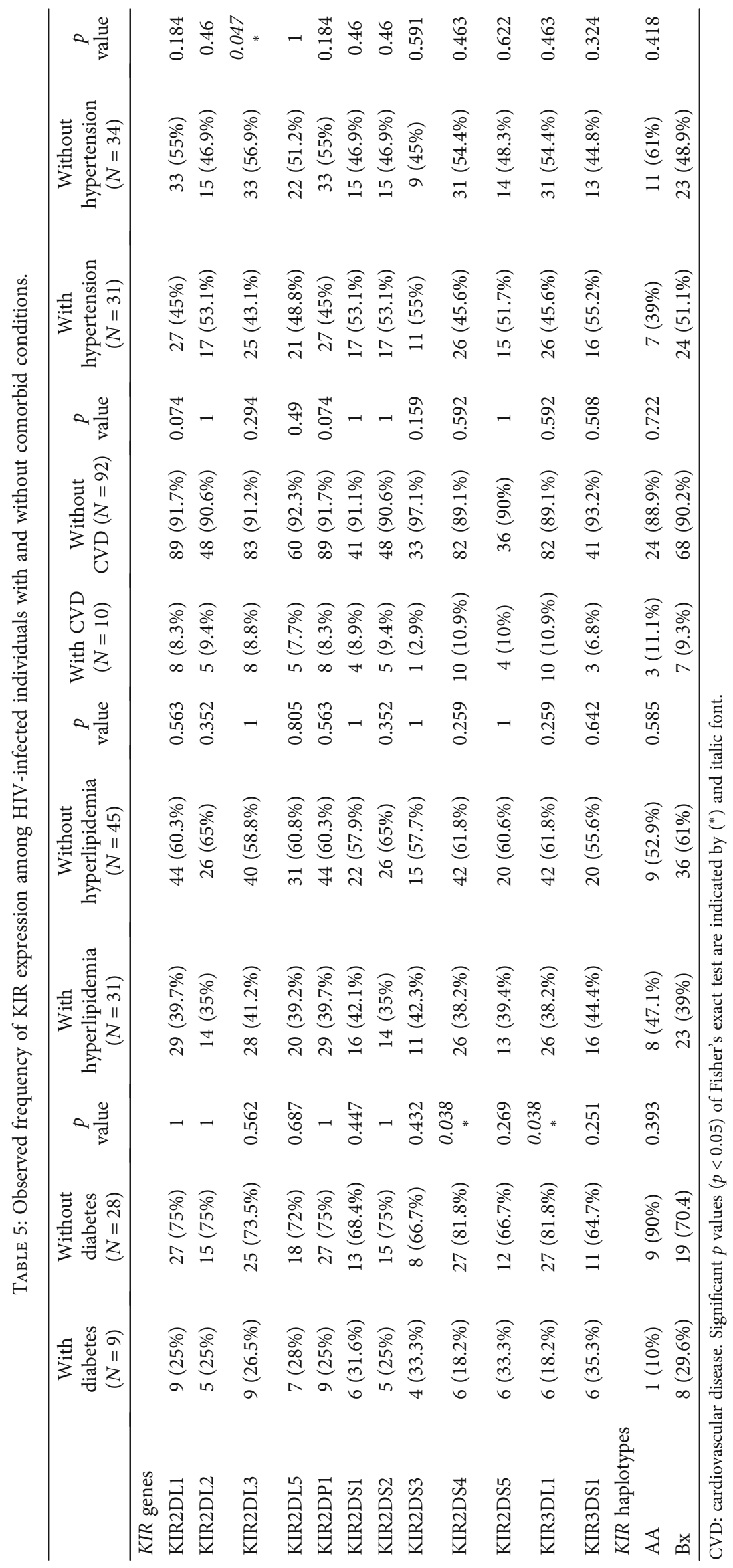


TABLE 6: The relationship between KIR genes and non-AIDS associated comorbid conditions.

\begin{tabular}{|c|c|c|c|c|c|c|c|c|}
\hline \multirow[t]{2}{*}{ KIR genes } & \multicolumn{2}{|l|}{ Diabetes } & \multicolumn{2}{|c|}{ Hyperlipidemia } & \multicolumn{2}{|c|}{ Hypertension } & \multicolumn{2}{|l|}{ CVD } \\
\hline & OR $(95 \% \mathrm{CI})$ & $p$ value & OR $(95 \% \mathrm{CI})$ & $p$ value & OR $(95 \% \mathrm{CI})$ & $p$ value & OR $(95 \% \mathrm{CI})$ & $p$ value \\
\hline \multicolumn{9}{|c|}{ Inhibitory KIRs } \\
\hline KIR2DL1 & 1 & - & $0.3(0.01-4.8)$ & 0.362 & $0.1(0.01-1.78)$ & 0.129 & $0.08(0.01-0.69)$ & 0.022 \\
\hline KIR2DL2 & $1.1(0.19-6.65)$ & 0.886 & $0.5(0.16-1.4)$ & 0.179 & $1.3(0.4-4.29)$ & 0.664 & $1.04(0.25-4.31)$ & 0.954 \\
\hline KIR2DL3 & 1 & - & $0.7(0.12-4.29)$ & 0.721 & $0.08(0.006-0.99)$ & 0.05 & $0.2(0.03-1.46)$ & 0.113 \\
\hline KIR2DL5 & $2.3(0.3-17.48)$ & 0.422 & $1.3(0.42-3.97)$ & 0.651 & $1.9(0.51-7.12)$ & 0.341 & $0.6(0.13-2.6)$ & 0.476 \\
\hline KIR3DL1 & $0.14(0.01-2.05)$ & 0.151 & $0.4(0.08-2.06)$ & 0.272 & $0.6(0.1-3.01)$ & 0.493 & 1 & - \\
\hline \multicolumn{9}{|c|}{ Activating KIRs } \\
\hline KIR2DS1 & $2.1(0.32-13.7)$ & 0.44 & $1.4(0.49-3.8)$ & 0.555 & $2.7(0.76-9.35)$ & 0.124 & $0.8(0.2-3.29)$ & 0.759 \\
\hline KIR2DS2 & $1.1(0.19-6.64)$ & 0.886 & $0.5(0.16-1.4)$ & 0.179 & $1.3(0.4-4.29)$ & 0.664 & $1.04(0.25-4.31)$ & 0.954 \\
\hline KIR2DS3 & $2.4(0.39-15.42)$ & 0.34 & $1.2(0.42-3.54)$ & 0.715 & $1.6(0.43-5.68)$ & 0.501 & $0.2(0.02-1.83)$ & 0.11 \\
\hline KIR2DS4 & $0.14(0.01-2.05)$ & 0.151 & $0.4(0.08-2.06)$ & 0.272 & $0.6(0.1-3.01)$ & 0.493 & 1 & - \\
\hline KIR2DS5 & $2.3(0.36-14.35)$ & 0.377 & $1.03(0.37-2.85)$ & 0.962 & $1.8(0.53-5.75)$ & 0.355 & $1.04(0.25-4.26)$ & 0.962 \\
\hline KIR3DS1 & $2.5(0.39-15.45)$ & 0.34 & $1.7(0.6-4.83)$ & 0.32 & $2.6(0.75-9.15)$ & 0.131 & $0.6(0.14-2.67)$ & 0.507 \\
\hline \multicolumn{9}{|c|}{ Pseudogenes } \\
\hline KIR2DP1 & 1 & - & $0.3(0.01-4.77)$ & 0.362 & $0.14(0.01-1.78)$ & 0.129 & $0.08(0.01-0.69)$ & 0.022 \\
\hline
\end{tabular}

CVD: cardiovascular disease; OR: odds ratio; 95\% CI: 95\% confidence interval. We adjusted for age, sex, and family history of comorbid condition. For hypertension and CVD, we also adjusted for smoking and alcohol use. We applied Bonferroni correction, and thus, $p$ values of $<0.004$ were considered significant.

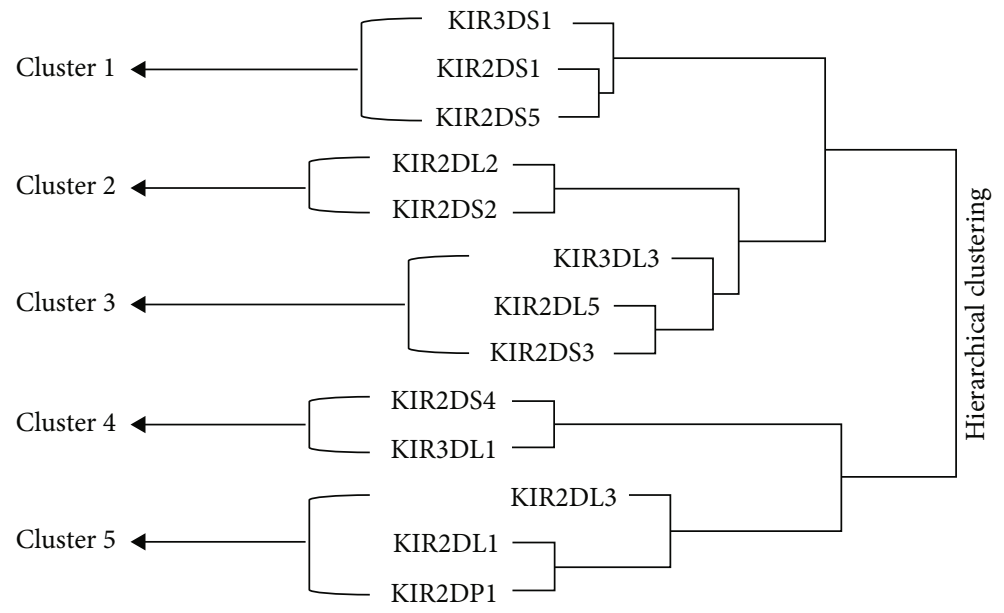

FIGURE 1: Clustering analysis of KIR genes among study participants.

different among participants with CVD who were $>40$ years of age. We detected a borderline significant difference $(p=0.053)$ in the expression of KIR2DL1 and KIR2DP1 among males with CVD (data not shown). Moreover, there was no significant difference in the expression of $\mathrm{Bx}$ or $\mathrm{AA}$ haplotypes among participants with or without any of the conditions above (Table 5). Collectively, these results suggest that KIR3DL1 and KIR2DS4 as well as KIR2DL3 can be associated with diabetes and hypertension, respectively, among people living and aging with HIV. These data also suggest that KIR2DL1, KIR2DP1, and KIR2DS3 are significantly associated with CVD among participants $>40$ years in our cohort.

We then analyzed in a multivariate analysis the relationship between KIR genes expression and the risk of having non-AIDS comorbidities. We adjusted for age, sex, and fam- ily history for the respective comorbid condition; for hypertension and CVD, we also adjusted for additional risk factors, specifically smoking and alcohol use. Our results showed that participants expressing KIR2DL1 and KIR2DP1 were significantly less likely to have CVD (OR 0.08; 95\% CI, 0.01-0.69; $p=0.022$ ) (Table 6). However, we did not detect any significant difference following post hoc comparisons.

3.4. KIR Clusters and Comorbidities. Based on the KIR genes clustering analysis, we identified five KIR clusters: Cluster 1, Cluster 2, Cluster 3, Cluster 4, and Cluster 5 (Figure 1). Cluster 1 corresponds to the telomeric cluster containing KIR3DS1, KIR2DS1, and KIR2DS5. We detected significant difference in the expression of Cluster 4 between diabetic and non-diabetic participants $(p=0.038)$ and in the expression of Cluster 5 between hypertensives and normotensives 


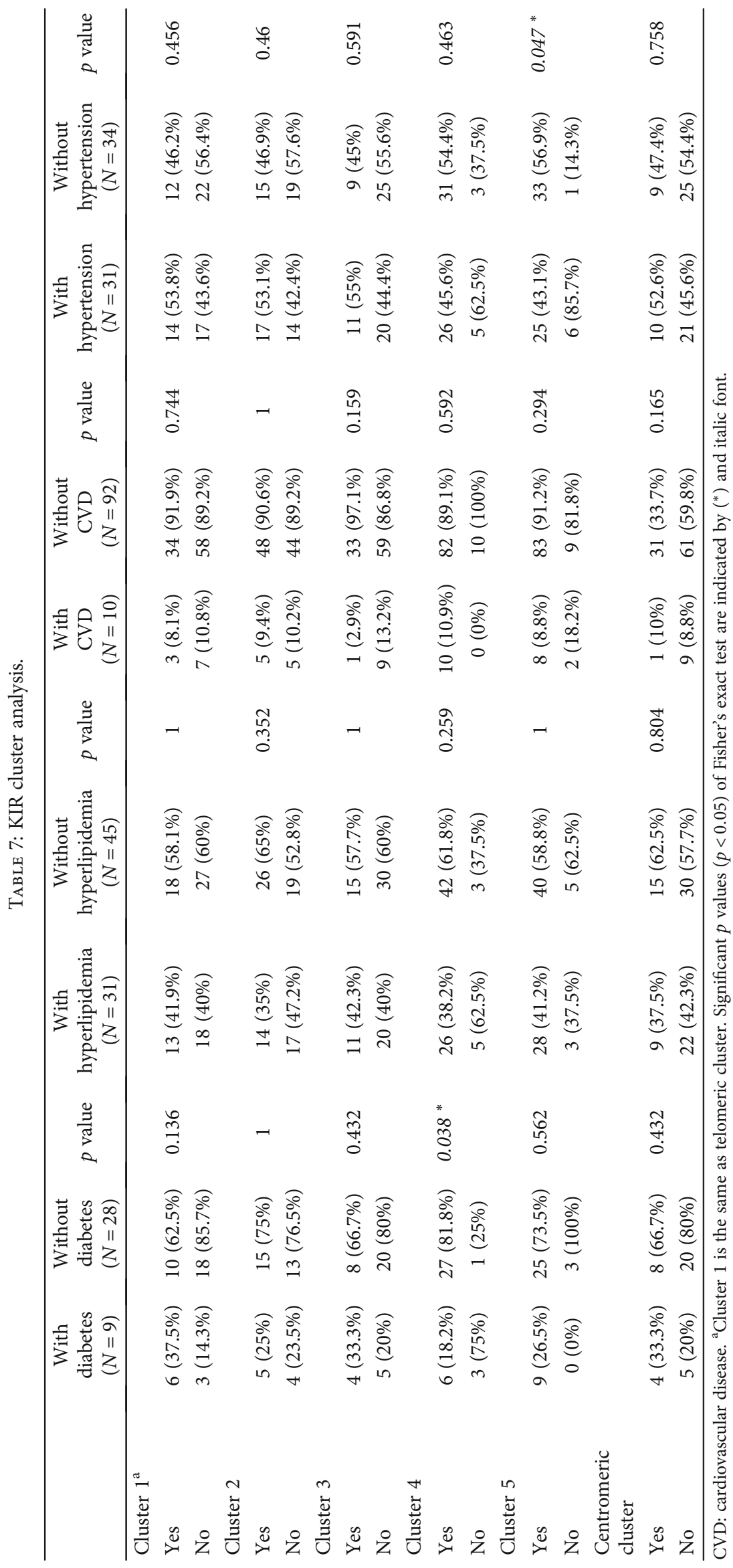


TABLE 8: The relationship between KIR clusters and non-AIDS associated comorbid conditions.

\begin{tabular}{lcccccccc}
\hline & \multicolumn{2}{c}{ Diabetes } & \multicolumn{2}{c}{ Hyperlipidemia } & \multicolumn{2}{c}{ Hypertension } & \multicolumn{2}{c}{ CVD } \\
& OR (95\% CI) & $p$ value & OR (95\% CI) & $p$ value & OR (95\% CI) & $p$ value & OR (95\% CI) & $p$ value \\
\hline KIR clusters & & & & & & & & \\
Cluster $1^{\mathrm{a}}$ & $2.7(0.44-16.45)$ & 0.283 & $1.27(0.45-3.58)$ & 0.649 & $1.7(0.52-5.82)$ & 0.373 & $0.8(0.18-3.6)$ & 0.786 \\
Cluster 2 & $1.14(0.19-6.65)$ & 0.886 & $0.5(0.16-1.4)$ & 0.179 & $1.3(0.4-4.29)$ & 0.664 & $1.04(0.25-4.31)$ & 0.954 \\
Cluster 3 & $2.4(0.39-15.53)$ & 0.34 & $1.2(0.41-3.54)$ & 0.715 & $1.6(0.43-5.68)$ & 0.501 & $0.2(0.02-1.83)$ & 0.154 \\
Cluster 4 & $0.14(0.01-2.04)$ & 0.151 & $0.4(0.08-2.06)$ & 0.272 & $0.6(0.1-3)$ & 0.493 & 1 & - \\
Cluster 5 & 1 & - & $0.7(0.12-4.29)$ & 0.721 & $0.08(0.006-0.99)$ & 0.05 & $0.2(0.03-1.46)$ & 0.113 \\
Centromeric cluster & $2.4(0.39-15.43)$ & 0.34 & $0.9(0.29-2.63)$ & 0.82 & $1.2(0.32-4.37)$ & 0.811 & $0.2(0.02-2.13)$ & 0.195 \\
\hline
\end{tabular}

CVD: cardiovascular disease; OR: odds ratio; 95\% CI: 95\% confidence interval. ${ }^{a}$ Cluster 1 is the same as telomeric cluster. We adjusted for age, sex, and family history of comorbid condition. For hypertension and CVD, we also adjusted for smoking and alcohol use. We applied Bonferroni correction, and thus, $p$ values of $<0.004$ were considered significant.

$(p=0.047)$ (Table 7). We did not detect any significant difference between centromeric (Cent-A and Cent-B) and telomeric (Tel-A and Tel-B) clusters and any comorbid condition (data not shown). The multivariate analysis revealed that individuals expressing Cluster 5 have significantly reduced odds of hypertension (OR 0.08; 95\% CI: 0.006-0.99; $p=0.05$ ) (Table 8).

\section{Discussion}

PLHIV are at higher risk of developing non-AIDS associated comorbidities than the general population; moreover, the prevalence of these comorbidities among PLHIV increases with age $[6,8,11]$. Our data showed that the frequency of comorbid conditions was higher among HIV-positive individuals $>40$ years compared to those $\leq 40$ years of age (Abou Hassan et al. submitted manuscript). In accordance with previous reports from our group (Hammad et al. accepted manuscript JIDC) and worldwide [14, 49-52], the most commonly reported comorbidities among our study participants were hypertension and hyperlipidemia followed by CVD and diabetes.

A limited number of studies exists on the prevalence of chronic disease conditions in the Lebanese population. Recently, the prevalence of hypertension among Lebanese adults $(n=2014)$ was reported at $31 \%$. The former was higher among older participants and those with higher body mass index (BMI) or reported to have CVD [53]. The prevalence of diabetes mellitus in Lebanon $(n=17,832)$ was reported at $7.95 \%$. The prevalence of type 1 diabetes was estimated at $0.1 \%$ [54]. Studies on the prevalence of comorbidities among PLHIV in the Middle East and North Africa (MENA) region are currently lacking except for reports from Iran [55] whereby hyperglycemia was recently reported to be highly prevalent among a cohort of PLHIV. This study identified older age, male gender, higher BMI, and prolonged duration of HIV infection as associated risk factors [55]. Recently, diabetes mellitus followed by dyslipidemia and hypertension was predominantly reported among a small group of HIV-infected individuals in western Saudi Arabia with age being a major risk factor [56]. Consequently, more studies are needed to determine the prevalence of non-AIDS associated comorbid conditions among PLHIV in the MENA region.

We observed high frequency of KIR2DS4, KIR2DL1, KIR2DP1, and KIR3DL1 genes among our study participants. Our results were similar to previously reported data in the region specifically among healthy Lebanese [48, 57], Iranian [58, 59], and Turkish adults [60]. Similar results were also reported in Southern Brazil [61, 62] and Eastern Han populations in China [63]. Moreover, the AA genotype profile was the most frequent among our study participants similar to previously reported data in healthy unrelated individuals in Lebanon [57], Turkey [60], Iran [58], and Tunisia [64].

Several studies reported on KIR genes expressions among individuals with chronic conditions such as diabetes [42], hypertension [44], malignancies (colorectal cancer [65], biliary cancer [32], breast cancer [66], and leukemia $[67,68])$, and primary immunodeficiency disorders such as common variable immune deficiency (CVID) [69]. While the frequency of KIR genes did not differ between diabetics and healthy controls among the Chinese Han [70], Basque [71], and Saudi populations [72], the expression of KIR2DL3 was significantly different among British HIV-naïve children ( $<5$ years) with type 1 diabetes compared to healthy controls [73]. We report a significant difference in the frequency of KIR2DS4 and KIR3DL1 between diabetic and non-diabetic HIV-positive participants. A recent meta-analysis showed that the expressions of KIR2DL1, KIR2DL2, and KIR2DL5 were significantly associated with susceptibility to T1DM [42]. KIR2DL2 was associated with increased risk of type 1 diabetes whereas KIR2DL1 and KIR2DL5 decreased the risk of the latter. However, we did not detect any significant difference in the expression of these genes between diabetics and non-diabetics among our study participants. While our results showed a significant difference in the frequency of KIR2DL3 between hypertensive and normotensive HIVpositive individuals, Wang et al. reported a significant association between the expression of KIR2DS5 and decreased risk of hypertension [44]. While our data did not reveal any association between the expression of KIR genes and 
risk of non-AIDS comorbidities, this could be due to ethnicity, sample size, and the population under study. Previous studies compared the frequency of KIR genes between individuals with a chronic condition and healthy subjects, while we compared the expression of KIR genes among HIVinfected subjects with and without non-AIDS comorbid conditions.

Despite the increased expression of centromeric and telomeric KIR clusters among hypertensive compared to normotensive participants, there was no significant association between these clusters and non-AIDs comorbid conditions. This is probably due to our small sample size. A recent study in China showed that centromeric KIR cluster (KIR2DS2-2DL2-2DS3-2DL5) was significantly increased in children with B-cell acute lymphoblastic leukemia (B-ALL) compared to healthy controls and provided the first evidence that this gene cluster might increase the susceptibility to BALL in Chinese Han children [74]. This result suggests that KIR gene clusters might be a predisposing factor for susceptibility to diseases and warrants further investigation. To our knowledge, the relationship between KIR clusters among PLHIV and non-AIDS comorbid conditions was not previously explored.

Our study has several limitations. Our study lacks a control group of HIV-negative individuals; thus, we were unable to compare the frequencies of KIR genes between our cohort and HIV-naïve individuals. Our study is a cross-sectional study without historic clinical and medical data to assess the evolution of comorbid conditions and pertinent risk factors across time. Moreover, we were unable to investigate the interaction between KIRs and their putative human leukocyte antigen class I (HLA-I) ligand. The latter has been implicated in controlling HIV-disease progression [75-79] and in the control or the progression of other viral diseases including human influenza virus, viral hepatitis ( $\mathrm{HCV}$ and HBV), and human cytomegalovirus (HCMV) [26, 77].

\section{Conclusion}

Additional studies with larger populations are needed to elucidate the role of KIRs in susceptibility or resistance to nonAIDS comorbid conditions among PLHIV. Importantly, the burden of non-AIDS comorbidities among people living and aging with HIV is critically needed in the region. These studies are important for the proper management and care of comorbid conditions among PLHIV. Understanding the molecular mechanisms governing the genetic factors modulating living and aging with HIV should be prioritized for screening and intervention to prevent and mitigate multimorbidities among people living and aging with HIV.

\section{Data Availability}

The clinical, biological, and demographic data used to support the findings of this study are included in the article.

\section{Conflicts of Interest}

The authors declare that they have no competing interests.

\section{Authors' Contributions}

FFA performed laboratory experiments, data collection, and analysis and wrote part of the manuscript. $\mathrm{MBH}$ performed data analysis and contributed to writing. KA guided and contributed to the analysis. NMM designed the study, oversaw all aspects of the study, edited, and finalized the manuscript. All authors reviewed, edited, and approved the final manuscript.

\section{Acknowledgments}

We thank all our voluntary participants and Ms. Dina Martinos (LAU), Ms. Rita Wahab, and Ms. Maria Helayel (SIDC) for their help in recruitment of volunteers. We would also like to thank Dr. Souha Kanj Sharara and Dr. Jean Francois Jabbour (AUBMC) for their help in the recruitment process. We also thank Dr. Rami Mahfouz for allowing us to use his lab to perform KIR genotyping and Ms. Fatmeh Abbas for her assistance in the lab. This study is supported by the University Review Board at the American University of Beirut (Award A103785) and the Lebanese National Council for Scientific Research (Award A103074).

\section{References}

[1] K. Yoshimura, "Current status of HIV/AIDS in the ART era," Journal of Infection and Chemotherapy, vol. 23, no. 1, pp. 1216, 2017.

[2] J. T. Brooks, K. Buchacz, K. A. Gebo, and J. Mermin, "HIV infection and older Americans: the public health perspective," American Journal of Public Health, vol. 102, no. 8, pp. 15161526, 2012.

[3] E. Kaplan-Lewis, J. A. Aberg, and M. Lee, "Aging with HIV in the ART era," in Seminars in diagnostic pathology, Elsevier, 2017.

[4] F. Nakagawa, M. May, and A. Phillips, "Life expectancy living with HIV," Current Opinion in Infectious Diseases, vol. 26, no. 1, pp. 17-25, 2013.

[5] C. S. Autenrieth, E. J. Beck, D. Stelzle, C. Mallouris, M. Mahy, and P. Ghys, "Global and regional trends of people living with HIV aged 50 and over: estimates and projections for 20002020," PLoS One, vol. 13, no. 11, article e0207005-e, 2018.

[6] A. Calcagno, S. Nozza, C. Muss et al., "Ageing with HIV: a multidisciplinary review," Infection, vol. 43 , no. 5, pp. 509522, 2015.

[7] G. V. Escota, J. A. O’Halloran, W. G. Powderly, and R. M. Presti, "Understanding mechanisms to promote successful aging in persons living with HIV," International Journal of Infectious Diseases, vol. 66, pp. 56-64, 2018.

[8] P. W. Hunt, "HIV and aging: emerging research issues," Current opinion in HIV and AIDS, vol. 9, no. 4, pp. 302-308, 2014.

[9] M. Smit, K. Brinkman, S. Geerlings et al., "Future challenges for clinical care of an ageing population infected with HIV: a modelling study," Infectious Diseases, vol. 15, no. 7, pp. 810818, 2015.

[10] C. Chu, G. Umanski, A. Blank, P. Meissner, R. Grossberg, and P. A. Selwyn, "Comorbidity-related treatment outcomes among HIV-infected adults in the Bronx, NY," Journal of Urban Health, vol. 88, no. 3, pp. 507-516, 2011. 
[11] G. Guaraldi, G. Orlando, S. Zona et al., "Premature age-related comorbidities among HIV-infected persons compared with the general population," Clinical Infectious Diseases : an official publication of the Infectious Diseases Society of America, vol. 53, no. 11, pp. 1120-1126, 2011.

[12] B. Hasse, B. Ledergerber, H. Furrer et al., "Morbidity and aging in HIV-infected persons: the Swiss HIV cohort study," Clinical Infectious Diseases: an official publication of the Infectious Diseases Society of America, vol. 53, no. 11, pp. 1130-1139, 2011.

[13] C. T. Longenecker, C. Sullivan, and J. V. Baker, "Immune activation and cardiovascular disease in chronic HIV infection," Current Opinion in HIV and AIDS, vol. 11, no. 2, pp. 216225, 2016.

[14] J. Schouten, F. W. Wit, I. G. Stolte et al., "Cross-sectional comparison of the prevalence of age-associated comorbidities and their risk factors between HIV-infected and uninfected individuals: the AGEhIV cohort study," Clinical Infectious Diseases : an official publication of the Infectious Diseases Society of America, vol. 59, no. 12, pp. 1787-1797, 2014.

[15] T. S. Torres, S. W. Cardoso, L. S. Velasque et al., "Aging with HIV: an overview of an urban cohort in Rio de Janeiro (Brazil) across decades of life," The Brazilian Journal of Infectious Diseases, vol. 17, no. 3, pp. 324-331, 2013.

[16] J. K. Rockstroh, R. Mohr, G. Behrens, and U. Spengler, "Liver fibrosis in HIV," Current Opinion in HIV and AIDS, vol. 9, no. 4, pp. 365-370, 2014.

[17] K. E. Sherman, J. Rockstroh, and D. Thomas, "Human immunodeficiency virus and liver disease: an update," Hepatology, vol. 62, no. 6, pp. 1871-1882, 2015.

[18] D. E. Vance, M. Mugavero, J. Willig, J. L. Raper, and M. S. Saag, "Aging with HIV: a cross-sectional study of comorbidity prevalence and clinical characteristics across decades of life," Journal of the Association of Nurses in AIDS Care, vol. 22, no. 1, pp. 17-25, 2011.

[19] Y. C. Lo, M. Y. Chen, W. H. Sheng et al., "Risk factors for incident diabetes mellitus among HIV-infected patients receiving combination antiretroviral therapy in Taiwan: a case-control study," HIV Medicine, vol. 10, no. 5, pp. 302-309, 2009.

[20] J. Rosenthal and W. Tyor, "Aging, comorbidities, and the importance of finding biomarkers for HIV-associated neurocognitive disorders," Journal of NeuroVirology, vol. 25, no. 5, pp. 673-685, 2019.

[21] S. S. Spudich, "Immune activation in the central nervous system throughout the course of HIV infection," Current opinion in HIV and AIDS, vol. 11, no. 2, pp. 226-233, 2016.

[22] S. G. Deeks, S. R. Lewin, and D. V. Havlir, "The end of AIDS: HIV infection as a chronic disease," Lancet, vol. 382, no. 9903, pp. 1525-1533, 2013.

[23] B. C. Mpondo, "HIV infection in the elderly: arising challenges," Journal of Aging Research, vol. 2016, Article ID 2404857, 10 pages, 2016.

[24] K. V. Fitch, M. N. Feldpausch, and S. E. Looby, "Biomarkers and clinical indices of aging with HIV," HIV and Aging, vol. 42, pp. 47-58, 2017.

[25] K. S. Campbell and A. K. Purdy, "Structure/function of human killer cell immunoglobulin-like receptors: lessons from polymorphisms, evolution, crystal structures and mutations," Immunology, vol. 132, no. 3, pp. 315-325, 2011.

[26] M. A. Ivarsson, J. MichaÃ«lsson, and C. Fauriat, "Activating killer cell Ig-like receptors in health and disease," Frontiers in Immunology, vol. 5, article 184, 2014.
[27] L. Y. C. Takeshita, F. F. Gonzalez-Galarza, E. J. M. dos Santos et al., "A database for curating the associations between killer cell immunoglobulin-like receptors and diseases in worldwide populations," Database, vol. 2013, article bat021-bat, 2013.

[28] R. Rajalingam, "Diversity of killer cell immunoglobulin-like receptors and disease," Clinics in Laboratory Medicine, vol. 38, no. 4, pp. 637-653, 2018.

[29] A. A. Bashirova, R. Thomas, and M. Carrington, "HLA/KIR restraint of HIV: surviving the fittest," Annual Review of Immunology, vol. 29, no. 1, pp. 295-317, 2011.

[30] D. Roe, C. Vierra-Green, C. W. Pyo et al., "Revealing complete complex KIR haplotypes phased by long-read sequencing technology," Genes \& Immunity, vol. 18, no. 3, pp. 127-134, 2017.

[31] K. C. Hsu, S. Chida, D. E. Geraghty, and B. Dupont, “The killer cell immunoglobulin-like receptor (KIR) genomic region: gene-order, haplotypes and allelic polymorphism," Immunological Reviews, vol. 190, no. 1, pp. 40-52, 2002.

[32] M. Cornillet, H. Jansson, M. Schaffer et al., "Imbalance of genes encoding natural killer immunoglobulin-like receptors and human leukocyte antigen in patients with biliary cancer," Gastroenterology, vol. 157, no. 4, pp. 1067-1080.e9, 2019.

[33] S. Aranda-Romo, C. A. Garcia-Sepulveda, A. Comas-García et al., "Killer-cell immunoglobulin-like receptors (KIR) in severe A (H1N1) 2009 influenza infections," Immunogenetics, vol. 64, no. 9, pp. 653-662, 2012.

[34] C. de Rham, K. Hadaya, C. Bandelier, S. Ferrari-Lacraz, and J. Villard, "Expression of killer cell immunoglobulin-like receptors (KIRs) by natural killer cells during acute CMV infection after kidney transplantation," Transplant Immunology, vol. 31, no. 3, pp. 157-164, 2014.

[35] A. Podhorzer, M. Dirchwolf, A. Machicote et al., "The clinical features of patients with chronic hepatitis $\mathrm{C}$ virus infections are associated with killer cell immunoglobulin-like receptor genes and their expression on the surface of natural killer cells," Frontiers in Immunology, vol. 8, p. 1912, 2018.

[36] Z. Shan, J. Huang, Q. Liao et al., “Association of killer cell immunoglobulin-like receptors with spontaneous clearance of hepatitis $\mathrm{C}$ virus in the Chinese population," Transfusion, vol. 58, no. 4, pp. 1028-1035, 2018.

[37] K. Zwolińska, O. Błachowicz, T. Tomczyk et al., "The effects of killer cell immunoglobulin-like receptor (KIR) genes on susceptibility to HIV-1 infection in the Polish population," Immunogenetics, vol. 68, no. 5, pp. 327-337, 2016.

[38] L.-M. Yindom, M. Mendy, C. Bodimeade et al., "KIR content genotypes associate with carriage of hepatitis B surface antigen, e antigen and HBV viral load in Gambians," PLoS One, vol. 12, no. 11, article e0188307-e, 2017.

[39] S. Boulet, S. Sharafi, N. Simic et al., "Increased proportion of KIR3DS1 homozygotes in HIV-exposed uninfected individuals," AIDS, vol. 22, no. 5, pp. 595-599, 2008.

[40] V. R. Chavan, D. Chaudhari, S. Ahir, Z. Ansari, P. Mehta, and J. Mania-Pramanik, "Variations in KIR genes: a study in HIV1 serodiscordant couples," BioMed Research International, vol. 2014, Article ID 891402, 11 pages, 2014.

[41] B. J. M. Tallon, J. Bruneau, C. M. Tsoukas et al., "Time to seroconversion in HIV-exposed subjects carrying protective versus non protective KIR3DS1/L1 and HLA-B genotypes," PLoS One, vol. 9, no. 10, article e110480-e, 2014.

[42] S. Soltani, S. Mostafaei, S. Aslani, E. Farhadi, and M. Mahmoudi, “Association of KIR gene polymorphisms with 
type 1 diabetes: a meta-analysis," Journal of Diabetes \& Metabolic Disorders, vol. 19, no. 2, pp. 1777-1786, 2020.

[43] S. L. Liu, A. J. Zheng, and L. Ding, “Association between KIR gene polymorphisms and type 1 diabetes mellitus (T1DM) susceptibility: a PRISMA-compliant meta-analysis," Medicine (Baltimore), vol. 96, no. 52, article e9439, 2017.

[44] A. L. Wang, B. Jiang, X. Y. Qian, Q. Zhang, H. Peng, and Y. H. Zhang, "Association between killer cell immunoglobulin-like receptor 2DS5 gene with essential hypertension in the Chinese Han patients," International Journal of Immunogenetics, vol. 44, no. 6, pp. 343-349, 2017.

[45] on behalf of KIRIIND (KIR Infectious and Inflammatory Diseases) Collaborative Group, A. Tuttolomondo, D. di Raimondo et al., "HLA and killer cell immunoglobulin-like receptor (KIRs) genotyping in patients with acute ischemic stroke," Journal of Neuroinflammation, vol. 16, no. 1, p. 88, 2019.

[46] R. Dastmalchi, A. Farazmand, S. Noshad et al., "Polymorphism of killer cell immunoglobulin-like receptors (KIR) and their HLA ligands in Graves' disease," Molecular Biology Reports, vol. 41, no. 8, pp. 5367-5374, 2014.

[47] L. Denis, J. Sivula, P. A. Gourraud et al., "Genetic diversity of KIR natural killer cell markers in populations from France, Guadeloupe, Finland, Senegal and Reunion," Tissue Antigens, vol. 66, no. 4, pp. 267-276, 2005.

[48] N. M. Melhem, R. A. Mahfouz, K. Kreidieh et al., "Potential role of killer immunoglobulin receptor genes among individuals vaccinated against hepatitis B virus in Lebanon," World Journal of Hepatology, vol. 8, no. 29, pp. 1212-1221, 2016.

[49] J. J. Bigna, A. L. Ndoadoumgue, J. R. Nansseu et al., "Global burden of hypertension among people living with HIV in the era of increased life expectancy: a systematic review and meta-analysis," Journal of Hypertension, vol. 38, no. 9, pp. 1659-1668, 2020.

[50] J. L. Castilho, M. M. Escuder, V. Veloso et al., "Trends and predictors of non-communicable disease multimorbidity among adults living with HIV and receiving antiretroviral therapy in Brazil," Journal of the International AIDS Society, vol. 22, no. 1, article e25233, 2019.

[51] G. H. da Cunha, K. B. Franco, M. T. G. Galvão et al., "Diabetes mellitus in people living with HIV/AIDS: prevalence and associated risk factors," AIDS Care, vol. 32, no. 5, pp. 600-607, 2020.

[52] S. K. Masenga, F. Elijovich, J. R. Koethe et al., "Hypertension and metabolic syndrome in persons with HIV," Current Hypertension Reports, vol. 22, no. 10, pp. 1-8, 2020.

[53] M. Cherfan, J. Blacher, R. Asmar et al., "Prevalence and risk factors of hypertension: a nationwide cross-sectional study in Lebanon," Journal of Clinical Hypertension (Greenwich, Conn), vol. 20, no. 5, pp. 867-879, 2018.

[54] I. Bou-Orm and S. Adib, "Prevalence and clinical characteristics of diabetes mellitus in Lebanon: a national survey," Eastern Mediterranean Health Journal, vol. 26, no. 2, pp. 182-188, 2020.

[55] M. Rasoolinejad, E. Najafi, A. Hadadi et al., "Prevalence and associated risk factors of hyperglycemia and diabetes mellitus among HIV positive patients in Tehran, Iran," Infectious Disorders - Drug TargetsDisorders, vol. 19, no. 3, pp. 304-309, 2019.

[56] F. M. Farahat, Y. S. Alghamdi, A. F. Farahat et al., "The prevalence of comorbidities among adult people diagnosed with
HIV infection in a tertiary care hospital in western Saudi Arabia," Journal of Infection and Public Health., vol. 13, no. 11, pp. 1699-1704, 2020.

[57] R. Rayes, A. Bazarbachi, G. Khazen, A. Sabbagh, G. Zaatari, and R. Mahfouz, "Natural killer cell immunoglobulin-like receptors (KIR) genotypes in two Arab populations: will KIR become a genetic landmark between nations?," Molecular Biology Reports, vol. 35, no. 2, pp. 225-229, 2008.

[58] E. Ashouri, S. Farjadian, E. F. Reed, A. Ghaderi, and R. Rajalingam, "KIR gene content diversity in four Iranian populations," Immunogenetics, vol. 61, no. 7, pp. 483-492, 2009.

[59] E. Jamali, S. Barani, F. Yousefinejad, A. Ariafar, G. R. Talei, and A. Ghaderi, "KIRs gene content diversity in Iranians with urothelial bladder cancer," Molecular Biology Reports, vol. 45, no. 5, pp. 713-719, 2018.

[60] O. G. Ozturk, G. Polat, and U. Atik, "Diversity of killer cell immunoglobulin-like receptor genes in Southern Turkey," Molecular Biology Reports, vol. 39, no. 2, pp. 1989-1995, 2012.

[61] M. Jobim, P. Chagastelles, P. H. Salim et al., "Association of killer cell immunoglobulin-like receptors and human leukocyte antigen-C genotypes in South Brazilian with type 1 diabetes," Human Immunology, vol. 71, no. 8, pp. 799-803, 2010.

[62] M. Jobim, P. H. Salim, P. Portela et al., "Killer cell immunoglobulin-like receptor gene diversity in a Caucasian population of southern Brazil," International Journal of Immunogenetics, vol. 37, no. 2, pp. 83-89, 2010.

[63] X. Bao, T. Zhang, X. Wu et al., "Population-specific criterion to distinguish killer cell immunoglobulin-like receptor genotypes and haplotypes in a large Eastern Han population," Hla, vol. 95, no. 1, pp. 15-22, 2020.

[64] M. Bani, J. Seket, H. Kaabi et al., "Killer cell immunoglobulinlike receptor (KIR) locus profiles in the Tunisian population," Human Immunology, vol. 76, no. 5, pp. 355-361, 2015.

[65] P. Portela, J. Merzoni, J. D. Lindenau et al., "KIR genes and HLA class I ligands in a Caucasian Brazilian population with colorectal cancer," Human Immunology, vol. 78, no. 3, pp. 263-268, 2017.

[66] S. Y. Alomar, A. Alkhuriji, P. Trayhyrn, A. Alhetheel, A. AlJurayyan, and L. Mansour, "Association of the genetic diversity of killer cell immunoglobulin-like receptor genes and HLA-C ligand in Saudi women with breast cancer," Immunogenetics, vol. 69, no. 2, pp. 69-76, 2017.

[67] S. Tao, Y. He, L. Dong et al., "Associations of killer cell immunoglobulin-like receptors with acute myeloid leukemia in Chinese populations," Human Immunology, vol. 78, no. 3, pp. 269-273, 2017.

[68] S. Vejbaesya, P. Sae-Tam, A. Khuhapinant, and D. Srinak, "Killer cell immunoglobulin-like receptors in Thai patients with leukemia and diffuse large B-cell lymphoma," Human Immunology, vol. 75, no. 7, pp. 673-676, 2014.

[69] Y. Wang, T. Hwangpo, M. P. Martin et al., "Killer cell immunoglobulin-like receptors are associated with common variable immune deficiency pathogenesis," The Journal of Allergy and Clinical Immunology, vol. 138, no. 5, pp. 14951498, 2016.

[70] D. Zhi, C. Sun, S. K. Sedimbi, F. Luo, S. Shen, and C. B. Sanjeevi, "Killer cell immunoglobulin-like receptor along with HLA-C ligand genes are associated with type 1 diabetes in Chinese Han population," Diabetes/Metabolism Research and Reviews, vol. 27, no. 8, pp. 872-877, 2011. 
[71] I. Santin, G. P. de Nanclares, B. Calvo, A. Gaafar, L. Castaño, and J. R. Bilbao, "Killer cell immunoglobulin-like receptor $(K I R)$ genes in the Basque population: association study of KIR gene contents with type 1 diabetes mellitus," Human Immunology, vol. 67, no. 1-2, pp. 118-124, 2006.

[72] A. E. Osman, N. Eltayeb-ELSheikh, M. Mubasher et al., "Investigation of activating and inhibitory killer cell immunoglobulin-like receptors and their putative ligands in type 1 diabetes (T1D)," Human Immunology, vol. 77, no. 1, pp. 110-114, 2016.

[73] K. L. Mehers, A. E. Long, A. R. van der Slik et al., "An increased frequency of NK cell receptor and HLA-C group 1 combinations in early-onset type 1 diabetes," Diabetologia, vol. 54, no. 12, pp. 3062-3070, 2011.

[74] M. Jiang, X. Guo, L. Yuan, J. Gao, L. Huo, and Q. Li, "Killer cell immunoglobulin-like receptor gene cluster predisposes to susceptibility to B-cell acute lymphoblastic leukemia in Chinese children," International Journal of Clinical and Experimental Pathology, vol. 13, no. 3, pp. 536-542, 2020.

[75] S. Chaisri, N. Pabalan, S. Tabunhan, P. Tharabenjasin, N. Sankuntaw, and C. Leelayuwat, "Effects of the killer immunoglobulin-like receptor (KIR) polymorphisms on HIV acquisition: a meta-analysis," PLoS One, vol. 14, no. 12, p. e0225151, 2019.

[76] V. R. Chavan, Z. Ansari, P. Mehta, and J. Mania-Pramanik, "Distribution of killer immunoglobulin-like receptor genes in HIV infected long-term non-progressors from Mumbai, India," Indian Journal of Dermatology, Venereology, and Leprology, vol. 84, no. 2, p. 247, 2018.

[77] S. Jost and M. Altfeld, "Control of human viral infections by natural killer cells," Annual Review of Immunology, vol. 31, no. 1, pp. 163-194, 2013.

[78] S. Maruthamuthu, R. Rajalingam, K. Pandian et al., "Inhibitory natural killer cell receptor KIR3DL1 with its ligand Bw4 constraints HIV-1 disease among South Indians," AIDS, vol. 32, no. 18 , pp. 2679-2688, 2018.

[79] J. Mikulak, F. Oriolo, E. Zaghi, C. Di Vito, and D. Mavilio, "Natural killer cells in HIV-1 infection and therapy," AIDS (London, England), vol. 31, no. 17, pp. 2317-2330, 2017. 